\title{
Edwardsiella tarda Bacteremia with Psoas and Epidural Abscess as a Food-borne Infection: A Case Report and Literature Review
}

\author{
Kiyozumi Suzuki, Mitsuru Yanai, Yuta Hayashi, Hiromasa Otsuka, \\ Kimitoshi Kato and Masayoshi Soma
}

\begin{abstract}
:
Edwardsiella tarda is commonly isolated from aquatic environments and a variety of animals. We present the first case of E. tarda bacteremia with psoas and epidural abscess. The patient was a 65 -year-old woman with recurrent gastric cancer who had frequently consumed raw fish and grilled eel. She was successfully treated with antimicrobials and surgery. We also review reports published in English regarding E. tarda bacteremia in Japan and the experience at our hospital. On the basis of this review, we conclude that the major underlying disease leading to $E$. tarda bacteremia is malignancy and that the gastrointestinal tract is the most commonly affected organ. The overall mortality rate due to $E$. tarda bacteremia in our review was $38.1 \%$ (8/ 21). Although E. tarda bacteremia is rare, clinicians should be aware of this fatal food-borne infection.
\end{abstract}

Key words: Edwardsiella tarda bacteremia, psoas abscess, spinal epidural abscess, vertebral osteomyelitis, urinary tract infection, food-borne infection

(Intern Med 57: 893-897, 2018)

(DOI: 10.2169/internalmedicine.9314-17)

\section{Introduction}

Edwardsiella tarda, a member of the family Enterobacteriaceae, is a motile, facultatively anaerobic, Gram-negative rod that has been isolated from fresh and brackish water environments and a variety of animals (reptiles, amphibians, and fish, including catfish and eels) $(1,2)$. In humans, $E$. tarda is a rare pathogen that mainly causes gastroenteritis as a food-borne infection (3). Extraintestinal E. tarda infections have also been reported infrequently. The risk factors for extraintestinal infections include hepatobiliary diseases, malignancy, and diabetes mellitus (4).

We describe the first case of E. tarda bacteremia with psoas abscess, vertebral osteomyelitis, and spinal epidural abscess. The patient had frequently eaten raw fish and grilled eel. She was simultaneously diagnosed with recurrent gastric cancer presenting as peritoneal dissemination. We also review the published English case reports of E. tarda bacteremia in Japan and the experience at our hospital.

\section{Case Report}

A 65-year-old Japanese woman was admitted to our hospital with a fever, lumbago, and right groin pain that had persisted for 2 weeks. The patient had undergone total gastrectomy with cholecystectomy and splenectomy for advanced gastric cancer (stage IIIC) three years earlier followed by oral chemotherapy for one year and had since been relapse-free. She had chronic diarrhea. She drank 360 $\mathrm{mL}$ of sake (Japanese rice wine) per day. A dietary history revealed that she had frequently eaten sashimi (sliced raw fish) and grilled eel, including within a few days prior to the onset of symptoms.

On a physical examination, the patient appeared ill, with a temperature of $36.7^{\circ} \mathrm{C}$, pulse of 67 beats $/ \mathrm{min}$, blood pressure of $83 / 51 \mathrm{mmHg}$, and a respiratory rate of 18 breaths/ min. The cardiovascular and respiratory findings were unremarkable. There was bilateral costovertebral angle tenderness and knocking pain of the lumbar spine. There was no 


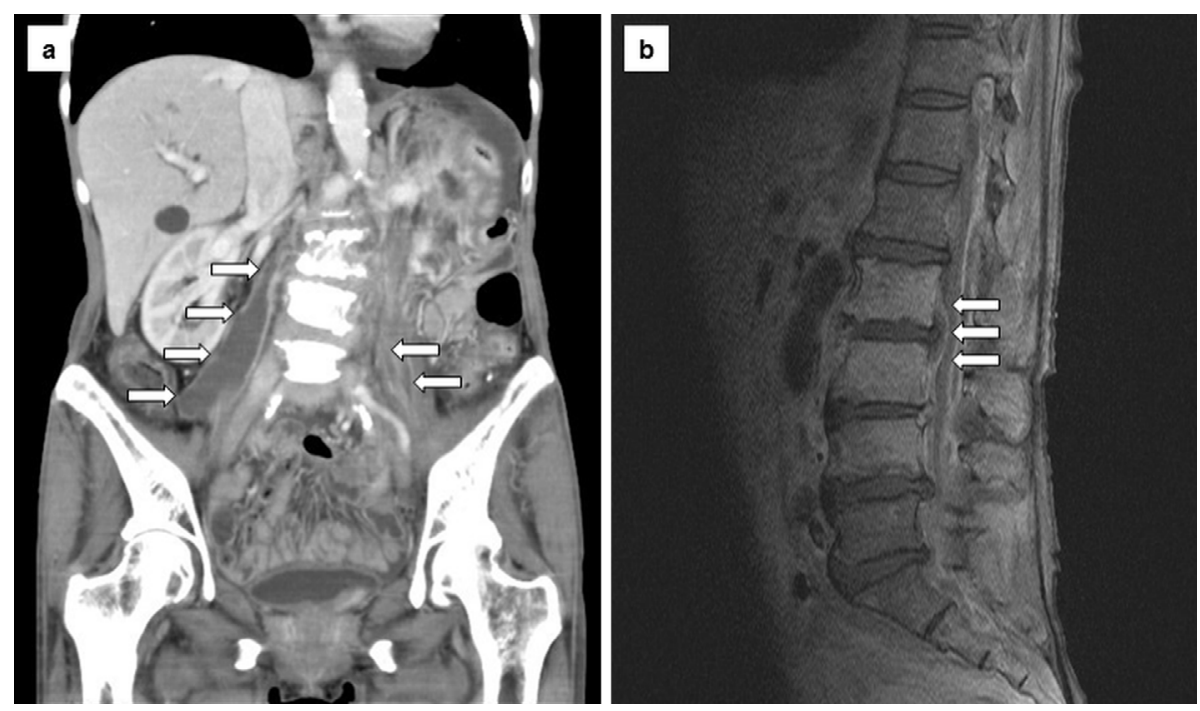

Figure. (a) A coronal contrast-enhanced computed tomography scan of the abdomen on admission showing a large, fluid-containing lesion with rim enhancement in the right psoas muscle and similar small lesions in the left psoas muscle (arrows). (b) T1-weighted magnetic resonance imaging of the lumbar spine with gadolinium enhancement on day 10 showing high intensity at the L2 to L4 vertebrae and epidural lesion with rim enhancement (arrows).

abdominal tenderness. An initial hematological investigation revealed a white blood cell count of $13.7 \times 10^{3} / \mu \mathrm{L}$ with $86 \%$ neutrophils, hemoglobin $9.9 \mathrm{~g} / \mathrm{dL}$, urea nitrogen $30.2 \mathrm{mg} / \mathrm{dL}$, creatinine $0.91 \mathrm{mg} / \mathrm{dL}$, albumin $1.7 \mathrm{~g} / \mathrm{dL}$, and C-reactive protein $19.8 \mathrm{mg} / \mathrm{dL}$. Liver enzyme levels were normal. Urinary sediment revealed a white blood cell count of $>100$ / high-powered field (HPF) and a red blood cell count of 10 to $19 / \mathrm{HPF}$.

Abdominal contrast-enhanced computed tomography (CT) revealed bilateral renal enlargement with perinephric stranding and a bilateral psoas abscess (Figure a). Magnetic resonance imaging (MRI) of the lumbar spine revealed vertebral osteomyelitis on the L2 to L4 vertebral bodies and disk space.

Empiric antimicrobial therapy with intravenous ceftriaxone ( 2 g every 24 hours) was started for urinary tract infection, psoas abscess, and vertebral osteomyelitis. Surgical drainage was not initially performed because of the high risk of postoperative complications due to hypoalbuminemia. The next day, the blood cultures became positive for a Gram-negative rod identified as Edwardsiella tarda by the RAISUS (Nissui Pharmaceutical, Tokyo, Japan) with susceptibility to ampicillin/sulbactam (minimum inhibitory concentration $\leq 4 \mu \mathrm{g} / \mathrm{mL})$, piperacillin/tazobactam $(\leq 8 \mu \mathrm{g} / \mathrm{mL})$, cefazoline $(\leq 2 \mu \mathrm{g} / \mathrm{mL})$, cefmetazole $(\leq 8 \mu \mathrm{g} / \mathrm{mL})$, cefotaxime $(\leq 1$ $\mu \mathrm{g} / \mathrm{mL})$, cefepime $(\leq 1 \mu \mathrm{g} / \mathrm{mL})$, aztreonam $(\leq 4 \mu \mathrm{g} / \mathrm{mL})$, meropenem $(\leq 1 \mu \mathrm{g} / \mathrm{mL})$, amikacin $(\leq 8 \mu \mathrm{g} / \mathrm{mL})$, and levofloxacin $(\leq 1 \mu \mathrm{g} / \mathrm{mL})$ and resistance to gentamicin $(>8 \mu \mathrm{g} / \mathrm{mL})$ and trimethoprim/sulfamethoxazole $(>80 \mu \mathrm{g} / \mathrm{mL})$. The organism was also isolated from urine culture on the day of admission but not from stool culture.

On day 5, based on the susceptibility testing, treatment with ceftriaxone was changed to intravenous cefmetazole (1 g every 6 hours). On day 24, the patient underwent surgical drainage and discectomy because the bilateral psoas abscess was gradually increasing in size and a spinal epidural abscess appeared (Figure b). No organisms grew from the abscess cultures. On day 38, she developed right lower abdominal pain. Abdominal CT revealed ileocecal diverticulitis, and cefmetazole was changed to meropenem ( $1 \mathrm{~g}$ every 8 hours). She underwent a barium enema and colonoscopy, which revealed multiple colon strictures. The pathological tissue revealed malignant cells in the membrane of the rectum above the peritoneal reflection, which were considered to be due to peritoneal dissemination of recurrent gastric cancer. Treatment with meropenem was continued, and her symptoms gradually improved. On day 85 , she was discharged after 12 weeks of intravenous antimicrobial therapy.

\section{Discussion}

To our knowledge, this is the first case of E. tarda bacteremia with psoas abscess, vertebral osteomyelitis, and spinal epidural abscess. In humans, E. tarda infections can be divided into two broad categories: gastrointestinal and extraintestinal. In $>80 \%$ of all cases reported, E. tarda is cultured from fecal specimens from patients in either the symptomatic or the asymptomatic carrier state (2). However, severe extraintestinal infections have also been reported, including bacteremia, wound infection, necrotizing fasciitis, hepatobiliary infection (liver abscess, cholecystitis), meningitis, osteomyelitis, urinary tract infection, endocarditis, tuboovarian abscess, salpingitis, brain abscess, and empyema (1-10). Historically, this organism is commonly found in both fresh and brackish water environments and in a variety of animals (reptiles, amphibians, and fish, includ- 


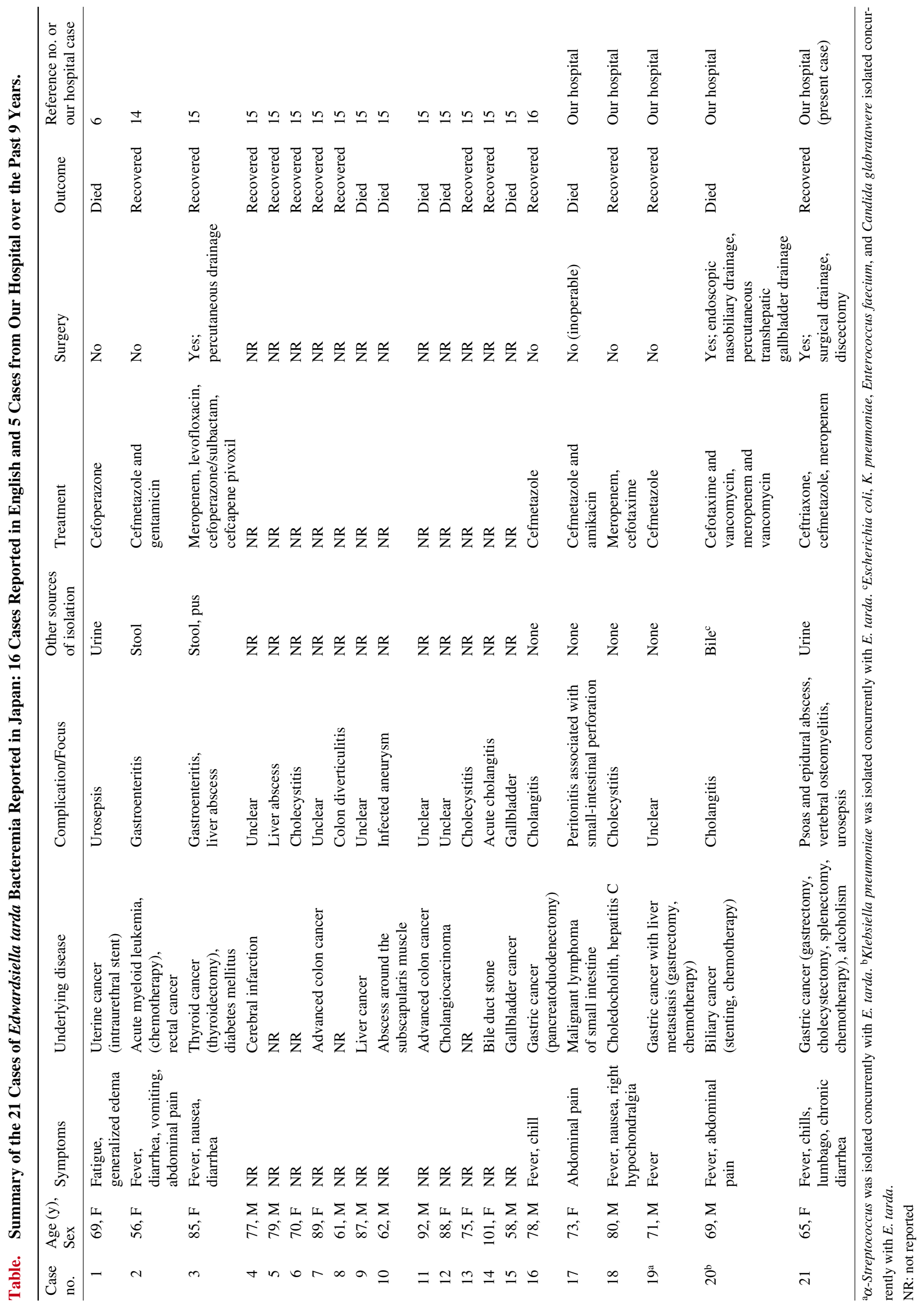


ing catfish and eels), and it can also cause disease in these animals (1-3). E. tarda has been isolated from $72 \%$ of farmcultured eels in the Republic of Korea (11). In contrast, $E$. tarda is not a member of the normal human flora, being isolated from only $0.0073 \%$ of fecal specimens from healthy Japanese (12). Therefore, E. tarda infection is considered to be food-borne, transmitted to humans by ingestion of contaminated food such as raw seafood (3).

Risk factors for E. tarda infection are wounding in aquatic environments, exposure to infected animals, dietary habits, and chronic underlying conditions (2). Aeromonas species (such as Aeromonas hydrophila) and Vibrio vulnificus are also known as aquatic pathogens. Aeromonas spp. are most commonly isolated from warm fresh and brackish waters, whereas $V$. vulnificus is most commonly isolated from brackish and marine environments (13). In humans, these pathogens can cause serious infections, such as bacteremia and necrotizing fasciitis, following ingestion of raw seafood or aquatic injury or exposure. Most patients infected with these pathogens are immunocompromised hosts, notably those with liver cirrhosis. Therefore, the clinical characteristics of E. tarda human infections and the environmental risks are similar to those of Aeromonas spp. and V. vulnificus.

In our patient, E. tarda was isolated from both blood and urine cultures. The patient did not have close contact with domestic or wild animals or any marine exposure prior to the onset of infection. However, she had frequently eaten sashimi and grilled eel. Therefore, the organism may have caused gastrointestinal colonization through the patient's ingestion of raw fish and grilled eels and may have resulted in retrograde urinary tract infection (6), although a stool culture was negative for the pathogen.

In previous reports, the major underlying conditions in $E$. tarda bacteremia were hepatobiliary diseases (liver cirrhosis, gallbladder stones, and ethanol abuse), malignancy (hepatobiliary and gastrointestinal tract), and iron overload states (sickle cell disease, leukemia, and neonatal state) (2, 3). Although E. tarda bacteremia is a rare complication $(<5 \%)$, the mortality rate is high, at nearly $50 \%$ (1-3). E. tarda is usually susceptible to most antimicrobials for Gram-negative bacteria except colistin and polymyxin $\mathrm{B}(1,2)$. The most frequently reported geographical area of E. tarda bacteremia cases is Japan, followed by the United States and the Republic of China (3). Japan has one of the world's highest rates of seafood consumption. We reviewed all case reports of E. tarda bacteremia in Japan published in English using PubMed. To date, 16 such cases have been reported (6, 14-16). In addition, our hospital encountered five cases of E. tarda bacteremia from 2008 to 2016 (Table). The median age of the 21 patients with E. tarda bacteremia was 75 years (range, 56-101 years), and 11 patients were men (52.4\%). Almost all patients with E. tarda bacteremia have significant underlying diseases; the major underlying disease was malignancy [13 of $17(76.5 \%)$, and the gastrointestinal tract was the most commonly affected organ [7 of
$13(53.8 \%)$, followed by the hepatobiliary tract [4 of 13 (30.8\%)]. Three patients required surgical treatment (one case was inoperable), and the overall mortality in our review was $38.1 \%$ ( 8 of 21 cases). Three patients (cases 16,19 , and 21 in Table) had previously undergone gastrectomy. We speculate that gastrectomy may increase the risk of E. tarda bacteremia, as it increases the risk of typhoid fever, due to a reduction of gastric acid secretion (17). In our patient, postgastrectomy state and the undiagnosed recurrent gastric cancer presenting as peritoneal dissemination probably contributed to the development of E. tarda bacteremia, and chronic alcohol consumption may also have contributed. Consequently, clinicians should consider underlying immunosuppressive conditions, including recurrent cancer, in patients with E. tarda bacteremia.

In conclusion, we have described the first case of E. tarda bacteremia with psoas abscess, vertebral osteomyelitis, and spinal epidural abscess, which was successfully treated with antimicrobials and surgery. Although E. tarda is a rare pathogen, it can cause fatal infections, like those caused by Aeromonas spp. and Vibrio vulnificus. Avoidance of consumption of raw or undercooked food is a simple measure to prevent fatal food-borne infections, and it is prudent for clinicians to emphasize the importance of this, especially in patients at high risk.

\section{The authors state that they have no Conflict of Interest (COI).}

\section{Acknowledgement}

We are grateful to Dr. Yuji Hirai, associate professor at Juntendo University, Faculty of Medicine, for his participation in helpful discussions on this manuscript.

\section{References}

1. Wilson JP, Waterer RR, Wofford JD Jr, Chapman SW. Serious infections with Edwardsiella tarda. A case report and review of the literature. Arch Intern Med 149: 208-210, 1989.

2. Janda JM, Abbott SL. Infections associated with the genus Edwardsiella: the role of Edwardsiella tarda in human disease. Clin Infect Dis 17: 742-748, 1993.

3. Hirai Y, Asahata-Tago S, Ainoda Y, Fujita T, Kikuchi K. Edwardsiella tarda bacteremia. A rare but fatal water- and foodborne infection: Review of the literature and clinical cases from a single centre. Can J Infect Dis Med Microbiol 26: 313-318, 2015.

4. Wang IK, Kuo HL, Chen YM, et al. Extraintestinal manifestations of Edwardsiella tarda infection. Int J Clin Pract 59: 917-921, 2005 .

5. Slaven EM, Lopez FA, Hart SM, Sanders CV. Myonecrosis caused by Edwardsiella tarda: a case report and case series of extraintestinal E. tarda infections. Clin Infect Dis 32: 1430-1433, 2001.

6. Tamada T, Koganemaru H, Matsumoto K, Hitomi S. Urosepsis caused by Edwardsiella tarda. J Infect Chemother 15: 191-194, 2009.

7. Nettles RE, Sexton DJ. Successful treatment of Edwardsiella tarda prosthetic valve endocarditis in a patient with AIDS. Clin Infect Dis 25: 918-919, 1997.

8. Golub V, Kim AC, Krol V. Surgical wound infection, tuboovarian abscess, and sepsis caused by Edwardsiella tarda: case reports and literature review. Infection 38: 487-489, 2010.

9. Mizunoe S, Yamasaki T, Tokimatsu I, et al. A case of empyema 
caused by Edwardsiella tarda. J Infect 53: e255-e258, 2006.

10. Takeuchi H, Fujita Y, Ogawa H, et al. Multiple brain abscesses in neonate caused by Edwardsiella tarda: case report. Neurol Med Chir (Tokyo) 49: 85-89, 2009.

11. Joh SJ, Kim MJ, Kwon HM, Ahn EH, Jang H, Kwon JH. Characterization of Edwardsiella tarda isolated from farm-cultured eels, Anguilla japonica, in the Republic of Korea. J Vet Med Sci 73: 7 11, 2011.

12. Onogawa T, Terayama T, Zen-yoji H, Amano Y, Suzuki K. Distribution of Edwardsiella tarda and hydrogen sulfide-producing Escherichia coli in healthy persons. Kansenshougaku Zasshi (J Jpn Assoc Infect Dis) 50: 10-17, 1976 (in Japanese).

13. Diaz JH. Skin and soft tissue infections following marine injuries and exposures in travelers. J Travel Med 21: 207-213, 2014.

14. Funada H, Kameoka J, Machi T, Matsuda T. Edwardsiella tarda septicemia complicating acute leukemia. Jpn J Med 27: 325-328, 1988.

15. Ohara Y, Kikuchi O, Goto T, et al. Successful treatment of a patient with sepsis and liver abscess caused by Edwardsiella tarda. Intern Med 51: 2813-2817, 2012.

16. Nishida K, Kato T, Yuzaki I, Suganuma T. Edwardsiella tarda bacteremia with metastatic gastric cancer. IDCases 5: 76-77, 2016.

17. Howden CW, Hunt RH. Relationship between gastric secretion and infection. Gut 28: 96-107, 1987.

The Internal Medicine is an Open Access article distributed under the Creative Commons Attribution-NonCommercial-NoDerivatives 4.0 International License. To view the details of this license, please visit (https://creativecommons.org/licenses/ by-nc-nd/4.0/).

(C) 2018 The Japanese Society of Internal Medicine Intern Med 57: 893-897, 2018 